\title{
EFECTOS DEL ACOPLAMIENTO \\ INTRAMOLECULAR EN LAS RESPUESTAS \\ OPTICAS DE SISTEMAS DE DOS NIVELES
}

\section{INTRAMOLECULAR COUPLING EFFECTS IN OPTICAL RESPONSES OF TWO-LEVEL SYSTEMS}

\section{José Luis Paz ${ }^{*}$, Eleana Ruiz-Hinojoza ${ }^{1}$, Ysaías Alvarado $^{2}$, Luis Lascano ${ }^{1}$ \& Cesar Costa-Vera ${ }^{1}$}

Recibido: 21 de febrero 2020 / Aceptado: 27 de abril 2020

Publicado en línea: 28 de abril 2020

DOI: 10.26807/ia.v8i2.137

Palabras claves: Acoplamiento Intramolecular, Adiabático, Diabático, Ecuaciones de Bloch ópticas Keywords: Intramolecular Coupling, Adiabatic, Diabatic, Optical Bloch equations

\section{RESUMEN}

Se evaluaron los efectos del acoplamiento intramolecular en las propiedades ópticas no lineales de colorantes orgánicos inmersos en un reservorio térmico empleando espectroscopia de mezcla de cuatro ondas. Se modeló la molécula

\footnotetext{
1 Escuela Politécnica Nacional, Departamento de Física, Quito, Ecuador (*correspondencia: jlpaz@usb.ve; jose.pazr@epn.edu.ec, angyruizh@yahoo.es, luis.lascano@epn.edu.ec, cesar.costa@epn.edu.ec)

2 Laboratorio de Caracterización Molecular y Biomolecular (LCMB), Instituto Venezolano de Investigaciones Científicas, Zulia, Venezuela (alvaradoysaias@gmail.com)
} 
aislada como curvas de energía Born-Oppenheimer consistiendo de dos estados electrónicos descritos como potenciales armónicos, con mínimos desplazados en coordenadas nucleares y energía. Inclusión de un Hamiltoniano residual de correlación electrónica y/o spin orbita, genera la separación de las dos curvas de acuerdo a las reglas de cruce evitado. Se evaluaron los momentos dipolares, tanto permanentes como de transición, como función de los parámetros del acoplamiento. Se usaron ecuaciones de Bloch ópticas convencionales para el cálculo de las polarizaciones inducidas en la aproximación tensorial, y con ellas las propiedades ópticas no lineales. Los resultados muestran atenuación de la intensidad en las respuestas ópticas y modificaciones en su topología, consecuencia del acoplamiento intramolecular y tipo de transición óptica. Amplificaciones paramétricas y posibilidad de Suiches en las propiedades ópticas, surgen como consecuencia de la inserción de acoplamientos intramoleculares.

\section{ABSTRACT}

In this work, the effects of the vibronic coupling on the nonlinear optical properties of organic dyes immersed in a thermal bath with the use of the FourWave mixing spectroscopy are evaluated. The isolated molecule is modeled as energy curves of Born-Oppenheimer, consisting of two-electronic states described by harmonic potentials, with minima displaced in energy and nuclear coordinates. Inclusion of a residual Hamiltonian of electronic correlation and/or spin-orbit generates the separation of the two curves according to the rules of avoided crossing. The permanent and transition dipole moments as function of the intramolecular coupling parameters are evaluated. We use the conventional optical Bloch equations, for the calculation of the polarizations induced in the tensorial approach and with them, the nonlinear optical properties. The results show attenuation of the intensity in the optical responses and modifications in their topology, as a consequence of the intramolecular coupling and type of optical transition. Parametric amplifications and the possibility of Switches in the optical properties, arise as a consequence of the insertion of intramolecular couplings. 


\section{INTRODUCCIÓN}

El desarrollo teórico de las propiedades ópticas no lineales, permite establecer la relación entre las características del material (estructura, composición química, etc.) y las de la radiación incidente, facilitando la interpretación y la predicción de mediciones ópticas en función de otras propiedades del sistema (Christodoulides et al., 2010; Guangjun, 2013). Por ejemplo, el coeficiente de absorción y el índice de refracción no lineales, dependen de la susceptibilidad del sistema. Ésta a su vez es función del momento dipolar eléctrico (Boyd, 2008) del mismo, entre otros parámetros. Esto juega un papel importante en los procesos de producción de colorantes (Christodoulides et al., 2010; Kajzar et al., 1994) y en el diseño de nuevos materiales (Guangjun, 2013). Pese a su importancia, varios autores (Squitieri et al., 1994; Paz et al., 2016a) han estimado valores de respuestas ópticas no lineales con base en momentos dipolares eléctricos constantes, cuando éstos son fuertemente influenciados por fenómenos como el acoplamiento intramolecular (Castro et al., 2004; Dunn et al., 2015). El acopla- miento intramolecular, es la interacción entre el movimiento nuclear y el movimiento electrónico en un sistema poliatómico, lo que produce un desplazamiento de los niveles de energía de los estados electrónicos del material, y en consecuencia su distribución electrónica se ve afectada (Bersuker, 2006; Di Bartolo \& Goldberg, 1980; Azumi \& Matsuzaki, 1977). El acoplamiento intramolecular corrige las funciones de onda de la aproximación Born-Oppenheimer BO que describen el sistema alterando el momento dipolar eléctrico. Así, la polarización macroscópica del sistema se modifica (Castro et al., 2004; Cusati et al., 2004; Paz et al., 1994), lo que implicaría que existe un efecto mesurable del acoplamiento intramolecular en las propiedades ópticas del sistema. Las investigaciones relacionadas con el acoplamiento intramolecular, han explicado fenó menos como la aparición de las denominadas transiciones electrónicas "prohibidas" por simetría en espectros de absorción, o la inestabilidad molecular de sistemas con configuraciones en estados electrónicos degenerados a través del efecto Jahn-Teller (Bersu- 
ker, 2006). El campo de trabajo ha abarcado temas como la influencia del acoplamiento en la interpretación de imágenes de microscopía de efecto túnel de aniones de Fullereno (Squitieri et al., 1994), y de espectroscopia de efecto túnel de electrones inelásticos (Walczak, 2006), su relación con el potencial químico y como éste mejora la transferencia de carga intermolecular, así como el diseño de moléculas de spin alto (Iwahara et al., 2011). En el presente trabajo, se estudió la influencia del acoplamiento intramolecular en la intensidad y topología de las propiedades ópticas no lineales de sistemas moleculares, en presencia de un reservorio térmico e interactuando con señales de mezcla de cuatro ondas MCO no degenerada. De los resultados obtenidos se evidencian atenuaciones importantes de las propiedades ópticas, así como modificaciones topológicas con respecto a los parámetros que caracterizan el acoplamiento inducido. Los estudios realizados pretenden una mayor comprensión de los procesos multifotónicos desde la perspectiva de análisis de los momentos dipolares derivados en los estados adiabáticos.

\section{METODOLOGÍA}

Se considera un sistema de dos niveles con estructura interna vibracional en presencia de un reservorio térmico y de radiación intensa de acuerdo con una interacción de tipo dipolareléctrica y siguiendo una dinámica de acuerdo al formalismo de Liouville. Con teoría cuántica de relajación, se derivaron las ecuaciones maestras generalizadas para los elementos de la matriz de densidad sujeta a la aproximación Markoviana. Bajo consideraciones fenomenológicas, se generaron las ecuaciones ópticas de
Bloch EOB (Boyd, 2008), las cuales permiten describir la dinámica de coherencias y de diferencias poblacionales en los estados electrónicos considerados. Empleando estas ecuaciones, el sistema molecular aislado se trató cómo potenciales armónicos desplazados en energías y coordenadas nucleares. A través de Hamiltonianos residuales se inducen las adiabaticidades y con ellas se calculan los momentos dipolares permanentes y de transición en la base no-acoplada (García-Sucre et al., 1970; Fulton \& 
Gouterman, 1964; Teller, 1937). Se resuelven las ecuaciones de coherencia y de población, bajo tratamientos perturbativos, haciendo uso de transformaciones de Fourier. Para la espectroscopia de MCO se calcularon las polarizaciones inducidas y con ellas las susceptibilidades a tercer orden, evaluadas bajo consideraciones locales (Paz et al., 2013). Las propiedades ópticas se calcularon a través de las susceptibilidades no lineales (Bavli et al., 1990; Jagatap \& Meath, 2002). El estudio se realizó a través de modificaciones en la saturación del haz intenso incidente, parámetros de acoplamiento, magnitudes de los momentos dipolares permanentes y de transición y finalmente, consideraciones en el tipo de transición óptica entre los estados electrónicos adiabáticos.

\section{Teoría}

\section{Acoplamiento intramolecular}

Se describe la molécula aislada como un sistema de dos niveles referidos a estados electrónicos, cuyas curvas de energía potencial son representadas por osciladores armónicos desplazados en coordenadas nucleares -Qo y energía Vo (Figura 1).

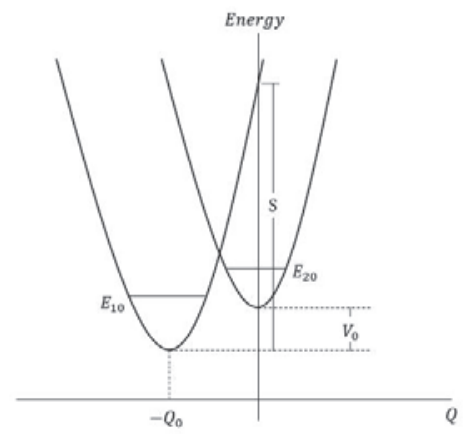

Figura 1. Representación diabática

Un Hamiltoniano residual de perturbación de correlación electrónica, o de tipo spin orbita, $\tilde{\mathrm{H}}$ acopla estos dos estados electrónicos. Las curvas previas pueden ser separadas de acuerdo con las reglas de cruzamiento evitado (Figura 2).

El Hamiltoniano asociado con el sistema molecular en presencia del acoplamiento intramolecular se define como $\mathrm{H}=\mathrm{H}_{0}+\mathrm{H}(\mathrm{r})$, donde $\mathrm{H}_{0}$ representa los dos osciladores desacoplados con estados vibracionales definidos, mientras que $\tilde{\mathrm{H}}(\mathrm{r})$ corresponde al término residual de perturbación. Las autofunciones de Ho son $\left|\psi_{1}\right\rangle$ y $\mid \psi_{2}$, con $\mathrm{H}_{0}\left|\psi_{1}\right\rangle=\mathrm{E}_{1 \mathrm{j}}\left|\psi_{1}\right\rangle$ y $\mathrm{H}_{0}\left|\psi_{2}\right\rangle=\mathrm{E}_{2 \mathrm{k}}\left|\psi_{2}\right\rangle$; $\{1,2\}$ representan los estados electrónicos y $\{\mathrm{j}, \mathrm{k}\}$ los estados vibracionales. 


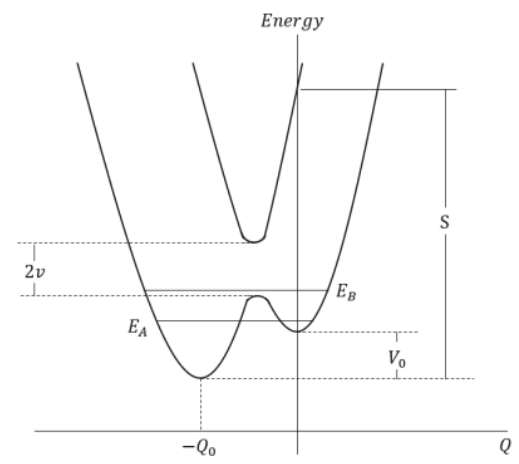

Figura 2. Representación adiabática

La inclusión de $\tilde{\mathrm{H}}(\mathrm{r})$ en el Hamiltoniano total hace $\mathrm{H}\left|\Psi_{\mathrm{A}}\right\rangle=\mathrm{E}_{\mathrm{A}}\left|\psi_{\mathrm{A}}\right\rangle$ y $\mathrm{H}\left|\Psi_{\mathrm{B}}\right\rangle=\mathrm{E}_{\mathrm{B}}\left|\Psi_{\mathrm{B}}\right\rangle$. En la BO se tiene $\left|\Psi_{\mathrm{A}}\right\rangle=\left|\chi_{1 j} \Psi_{1}\right\rangle \quad$ y $\left|\Psi_{\mathrm{B}}\right\rangle=\left|\chi_{2 \mathrm{k}} \Psi_{2}\right\rangle$. El acoplamiento intramolecular se representa por los elementos no diagonales del operador residual, dado por:

$$
\left\langle\chi_{1 \mathrm{j}} \psi_{1}|\tilde{\mathrm{H}}| \chi_{2 \mathrm{k}} \Psi_{2}\right\rangle=\mathrm{W}_{12}=\mathrm{v}\left\langle\chi_{1 \mathrm{j}} \mid \chi_{2 \mathrm{k}}\right\rangle
$$

y $v=\left\langle\psi_{1}|\tilde{H}| \psi_{2}\right\rangle$, definido como el coeficiente de acoplamiento, siendo $\left\langle\chi_{1 \mathrm{i}} \mid \chi_{2 \mathrm{k}}\right\rangle$ la integral de solapamiento. Resolviendo variacionalmente las energías de los estados adiabáticos, tenemos:

$E_{B}=\frac{1}{2}\left\{\left(E_{1 j}+E_{2 k}\right)+\left[\left(E_{1 j}-E_{2 k}\right)^{2}+4 v^{2} \mid\left\langle\chi_{1 j}\left|\chi_{2 k}\right|^{2}\right]^{1 / 2}\right\}\right.$

$E_{A}=\frac{1}{2}\left\{\left(E_{1 j}+E_{2 k}\right)-\left[\left(E_{1 j}-E_{2 k}\right)^{2}+4 v^{2}\left|\left\langle x_{1 j} \mid x_{2 k}\right\rangle\right|^{2}\right]^{1 / 2}\right\}$ y para las funciones de onda de $\mathrm{H}$, tenemos:

$$
\begin{aligned}
& \left|\Psi_{\mathrm{B}}\right\rangle=\left|\Psi_{\mathrm{B}}(\mathrm{r}, \mathrm{Q})\right\rangle=\mathrm{C}_{\mathrm{ij}}^{\mathrm{B}}\left[\Psi_{1}(\mathrm{r} ; \mathrm{Q}) \chi_{\mathrm{jij}}(\mathrm{Q})+\mathrm{A}_{\mathrm{jk}}^{\mathrm{B}} \Psi_{2}(\mathrm{r} ; \mathrm{Q}) \chi_{2 \mathrm{k}}(\mathrm{Q})\right] \\
& \left|\Psi_{\mathrm{A}}\right\rangle=\left|\Psi_{\mathrm{A}}(\mathrm{r}, \mathrm{Q})\right\rangle=\mathrm{C}_{\mathrm{ij}}^{\mathrm{A}}\left[\Psi_{1}(\mathrm{r} ; \mathrm{Q}) \chi_{\mathrm{ji}}(\mathrm{Q})-\mathrm{A}_{j \mathrm{k}}^{\mathrm{A}} \Psi_{2}(\mathrm{r} ; \mathrm{Q}) \chi_{2 \mathrm{k}}(\mathrm{Q})\right]
\end{aligned}
$$

donde r, Q representan las coordenadas electrónicas y nucleares, respectivamente.

$$
\begin{aligned}
& \mathrm{E}_{1 \mathrm{j}}=(\mathrm{j}+1 / 2) \hbar \omega_{0} ; \mathrm{E}_{2 \mathrm{k}}=(\mathrm{k}+1 / 2) \hbar \omega_{0}+\mathrm{V}_{0} \\
& \mathrm{C}_{1 \mathrm{j}}^{\mathrm{A}}=\left(1+\mathrm{A}_{\mathrm{jk}}^{\mathrm{A}}\right)^{-1 / 2} \quad \mathrm{C}_{1 \mathrm{j}}^{\mathrm{B}}=\left(1+\mathrm{A}_{\mathrm{jk}}^{\mathrm{B}}\right)^{-1 / 2} ; \\
& \mathrm{A}_{\mathrm{jk}}^{\mathrm{A}}=\left(\mathrm{E}_{1 \mathrm{j}}-\mathrm{E}_{\mathrm{A}}\right) /\left\langle\chi_{1 \mathrm{j}} \mid \chi_{2 \mathrm{k}}\right\rangle \mathrm{u} ; \\
& \mathrm{A}_{\mathrm{jk}}^{\mathrm{B}}=\left(\mathrm{E}_{1 \mathrm{j}}-\mathrm{E}_{\mathrm{B}}\right) /\left\langle\chi_{1 \mathrm{j}} \mid \chi_{2 \mathrm{k}}\right\rangle \mathrm{u} .
\end{aligned}
$$

La frecuencia de Bohr es $\omega_{0}=\left(E_{2 \mathrm{k}}-\mathrm{E}_{1 \mathrm{j}}\right) / \hbar$. En la base adiabática, se tiene los momentos dipolares de transición y permanentes de forma:

$$
\begin{aligned}
& \hat{\mu}_{\mathrm{AB}}=-\mathrm{C}_{1 \mathrm{j}}^{\mathrm{A}} \mathrm{C}_{1 \mathrm{j}}^{\mathrm{B}}\left[\mathrm{A}_{\mathrm{jk}}^{\mathrm{A}} \mathrm{A}_{\mathrm{jk}}^{\mathrm{B}} \mathrm{m}_{22}(0)+\mathrm{m}_{11}\left(-\mathrm{Q}_{0}\right)\right] \\
& -\mathrm{C}_{1 \mathrm{j}}^{\mathrm{A}} \mathrm{C}_{1 \mathrm{j}}^{\mathrm{B}} \mathrm{m}_{12}\left(-\mathrm{Q}_{0}\right)\left\langle\chi_{1 \mathrm{j}} \mid \chi_{2 \mathrm{k}}\right\rangle\left(\mathrm{A}_{\mathrm{jk}}^{\mathrm{B}}+\mathrm{A}_{\mathrm{jk}}^{\mathrm{A}}\right) \\
& \hat{\mu}_{\mathrm{rr}}=\left(\mathrm{C}_{1 \mathrm{j}}^{\mathrm{r}}\right)^{2}\left[\left(\mathrm{~A}_{\mathrm{jk}}^{\mathrm{r}}\right)^{2} \mathrm{~m}_{22}(0)+\mathrm{m}_{11}\left(-\mathrm{Q}_{0}\right)\right]- \\
& 2\left(\mathrm{C}_{1 \mathrm{j}}^{\mathrm{r}}\right)^{2} \mathrm{~m}_{12}\left(-\mathrm{Q}_{0}\right)\left\langle\chi_{1 \mathrm{j}} \mid \chi_{2 \mathrm{k}}\right\rangle\left(\mathrm{A}_{\mathrm{jk}}^{\mathrm{r}}\right)
\end{aligned}
$$

con $r=A$, B y donde $m_{11}$ y $m_{22}$ representan los momentos dipolares permanentes asociados a la base en la aproximación diabática. De la ecuación (4) es importante resaltar que el 
elemento no diagonal del momento dipolar diabático, es evaluado en - $Q_{0}$, de acuerdo con el principio de Franck-Condon (Guseinov et al., 2006). Podemos definir:

$$
\mathrm{m}_{\mathrm{ij}}(\mathrm{Q})=\int \psi_{\mathrm{i}}^{*}(\mathrm{r} ; \mathrm{Q}) \hat{\mathrm{m}} \psi_{\mathrm{j}}(\mathrm{r} ; \mathrm{Q}) \mathrm{d}^{3} \mathrm{r}
$$

El factor de solapamiento de los estados vibracionales $\left\langle\chi_{1 \mathrm{i}} \mid \chi_{2 \mathrm{k}}\right\rangle$ se define por la fórmula de Pekarian, (Di Bartolo \& Goldberg, 1980), así:

$$
\left\langle\chi_{1 \mathrm{j}} \mid \chi_{2 \mathrm{k}}\right\rangle=\frac{(-1)^{\mathrm{k}}}{(\mathrm{k} !)^{1 / 2}} \mathrm{~S}^{\mathrm{k} / 2} \exp \left(-\frac{\mathrm{S}}{4}\right) 2
$$

para $\mathrm{j}=0$

\section{Ecuaciones dinámicas ópticas de Bloch}

Para la interacción sistema-baño térmico, consideramos mecanismos de relajación en la aproximación Markoviana, mientras la interacción radiativa dipolar eléctrica, la consideramos de la forma:

$$
\partial_{t} \rho=M(t) \rho+R
$$

donde $\rho$ es la matriz densidad, $M(t)$ la matriz radiativa y $\mathrm{R}$ la correspondiente a la relajación.
$\rho=\left(\begin{array}{c}\rho_{\mathrm{AB}} \\ \rho_{\mathrm{BA}} \\ \rho_{\mathrm{D}}\end{array}\right), \mathrm{R}=\left(\begin{array}{c}0 \\ 0 \\ \frac{1}{T_{1}} \rho_{\mathrm{D}}^{(0)}\end{array}\right), \mathrm{d}=\mu_{\mathrm{BB}}-\mu_{\mathrm{AA}}$,

$$
M(t)=\left(\begin{array}{ccc}
0 & f(v) & \frac{i}{\hbar} \mu_{A B} E \\
f^{*}(v) & 0 & -\frac{i}{\hbar} \mu_{B A} E \\
-\frac{2 i}{\hbar} \mu_{A B} E & \frac{2 i}{\hbar} \mu_{B A} E & -\frac{1}{T_{1}}
\end{array}\right),
$$

con $\mathrm{f}(\mathrm{v})=\mathrm{i} \tilde{\omega}_{0}-\frac{1}{\mathrm{~T}_{2}}-\frac{\mathrm{i}}{\hbar} \mathrm{dE} ; \mathrm{T}_{1}$ y $\mathrm{T}_{2}$ son los tiempos de relajación longitudinal y transversal, respectivamente; el superíndice (0) indica los valores de equilibrio de la diferencia de población; E es la amplitud del campo total, $\mathrm{d}$ es la diferencia de momentos dipolares permanentes. La ecuación (6) se resuelve en el espacio de Fourier, considerando la mezcla de cuatro ondas. Dada la presencia de momentos dipolares permanentes de los estados adiabáticos, es necesario no considerar en los cálculos, aproximaciones de onda rotante (Paz et al. 1990). Este proceso de MCO implica la interacción de dos haces de frecuencias ópticas $\omega_{1}$ y $\omega_{2}$, y con direcciones de propagación $\overrightarrow{\mathrm{k}}_{1} \mathrm{y} \overrightarrow{\mathrm{k}}_{2}$. La señal de MCO se observa a la frecuencia $\omega_{3}=2 \omega_{1}-\omega_{2}$ (Paz et al., 
2013). Para efectos del cálculo de polarización $\mathrm{P}\left(\omega_{3}\right)$ son necesarias las componentes de Fourier de coherencia $\rho_{\mathrm{BA}}\left(\omega_{3}\right)$ y $\rho_{\mathrm{AB}}\left(\omega_{3}\right)$, y poblaciones en los estados adiabáticos $\rho_{\mathrm{AA}}\left(\omega_{3}\right)$ y $\rho_{\mathrm{BB}}\left(\omega_{3}\right)$, dada por

$P^{(3)}\left(\omega_{3}\right)=N\left[\rho_{A B}^{(3)}\left(\omega_{3}\right) \mu_{B A}+\rho_{B A}^{(3)}\left(\omega_{3}\right) \mu_{A B}+d \rho_{B B}^{(3)}\left(\omega_{3}\right)+\mu_{A A}\right]$

$\mathrm{N}$ es la concentración química de moléculas absorbentes y el superíndice (3) denota el orden de perturbación total. La ecuación (7) en términos de la susceptibilidad en la aproximación tensorial, se expresa como:

$$
\mathrm{P}^{(3)}\left(\omega_{3}\right)=\varepsilon_{0} \chi^{(3)}\left(\omega_{3}\right) \mathrm{E}_{1}^{2} \mathrm{E}_{2}^{*}
$$

donde $\varepsilon_{0}$ es la constante dieléctrica del material mientras que $\chi^{(3)}\left(\omega_{3}\right)$ es la susceptibilidad (compleja) de respuesta óptica. En este trabajo nos in- teresa la absorción como atenuación de la señal de MCO al propagarse en el medio. Considerando aproximaciones de envolvente suave para los campos clásicos, la ecuación de onda electromagnética en el presente tratamiento adquiere la forma:

$$
2 i k \partial_{z} E-k^{2} E+\omega^{2} / c^{2} E=-\mu_{0} \omega^{2} P
$$

Considerando aspectos propios de la electrodinámica clásica, es posible obtener el coeficiente de absorción, dado por:

$$
\alpha\left(\omega_{3}\right)=\frac{2 \pi \omega_{3}}{\eta\left(\omega_{3}\right) c} \operatorname{Im} \chi^{(3)}\left(\omega_{3}\right)
$$

donde $\eta\left(\omega_{3}\right)=\left[\eta_{0}^{2}+4 \pi \operatorname{Re} \chi^{(3)}\left(\omega_{3}\right)\right]^{1 / 2}$

es el índice de refracción no lineal, mientras $\eta_{0}$ es el índice de refracción del solvente puro.

\section{RESULTADOS}

La discusión del efecto causado por la interacción entre el movimiento nuclear y el electrónico, abarcará el empleo de transiciones entre niveles vibracionales distintos y una intensidad para el factor de acoplamiento alterable. Seleccionamos como ejemplo ilustrativo del modelo, el colorante orgánico Cloruro Verde Malaquita, de gran utilidad en comunicaciones ópticas y diseño de dispositivos optoelectrónicos. Nuestro cálculo supone 
un haz de bombeo a segundo orden de la MCO y por simplicidad, transiciones que se dan entre niveles vibracionales fundamentales.

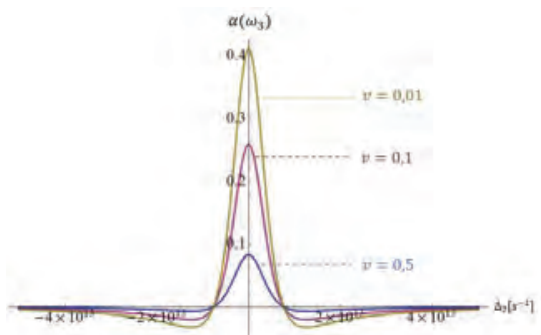

(a)

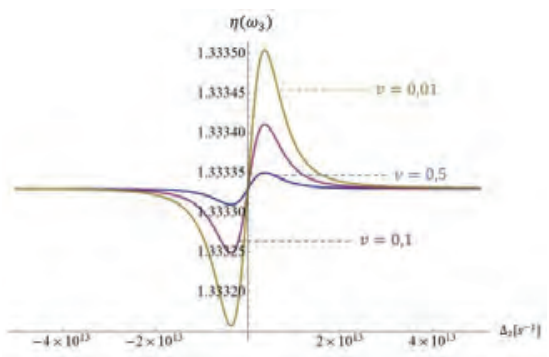

(b)

Figura 3. Perfiles de intensidad del coeficiente de absorción (a) e índice de refracción (b) del colorante orgánico Verde Malaquita con factores de acoplamiento $v=\{0,1-0,01-0,5\}$

La Figura 3(a) representa la variación del coeficiente de absorción no lineal, y Figura 3(b) la variación del índice de refracción, ambas como función del desintonizamiento en frecuencia del haz de prueba $\Delta_{2}\left(\mathrm{~s}^{-1}\right)$ pa- rametrizada por los valores de acoplamiento, en el caso de sistemas moleculares con momentos dipolares nulos en sus estados adiabáticos. Las Figuras 4(a) y 4(b) representan las mismas propiedades ópticas, pero en casos de dipolos permanentes no nulos.
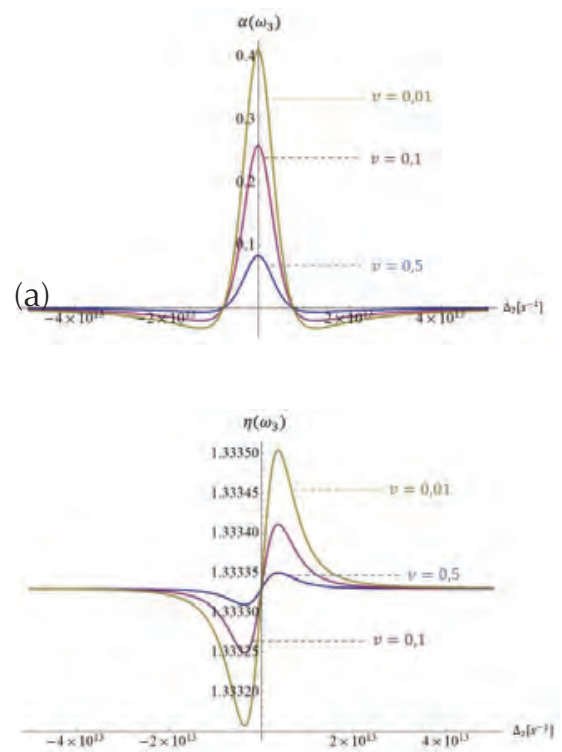

(b)

Figura 4. Perfiles de intensidad del coeficiente de absorción (a) e índice de refracción (b) del colorante orgánico Verde Malaquita con factores de acoplamiento

$$
v=\{0,1-0,01-0,5\}
$$

En las Figuras 3 y 4 , el perfil azul corresponde al sistema con $v=\{0,5\}$, el perfil rojo con $v=\{0,1\}$ y el perfil verde con $v=\{0,01\}$. 


\section{DISCUSIÓN}

Los perfiles absortivos (Figura 3(a) y Figura 4(a)), muestran la disminución de la intensidad de la propiedad óptica con el aumento del factor de acoplamiento, independientemente del valor de la diferencia de momentos dipolares permanentes. Sin embargo, en el perfil dispersivo, aumentos en el valor de acoplamiento, implica disminución en la intensidad de la propiedad óptica sólo en casos de momentos dipolares permanentes nulos. Comprendido, en el caso de momentos dipolares permanentes no nulos, la intensidad de la propiedad óptica (dispersión) pasa por un máximo localizado para el valor intermedio del acoplamiento intramolecular. Al comparar los dos perfiles absortivos, en el caso de valor nonulo para la diferencia de dipolos permanentes, la propiedad óptica alcanza un mayor valor de intensidad para un factor de acoplamiento mínimo. En ambos casos, valores de momentos dipolares permanentes nulos y no nulos, se nota en regiones cercanas a la resonancia un valor negativo de la absorción, consecuencia posiblemente de un proceso de amplificación paramétrica sintonizable con el colorante orgánico. A medida que el factor de acoplamiento, $v$, se aproxima a cero, menor es el acoplamiento entre los movimientos nuclear y electrónico de las moléculas en sus propiedades macroscópicas. Se conoce que $v$ juega un papel fundamental en la emisión de luz de una molécula al estimular transiciones no radiativas. Por tanto, un factor de acoplamiento pequeño, está relacionado con la anulación de estas transiciones. El factor $v$, además está asociado a las desviaciones geométricas en una molécula, lo que se conoce como el efecto pseudo-Jahn-Teller (Grochala \& Hoffmann, 2000). Es importante destacar que en la Figura 3(b) para un valor de factor de acoplamiento $v=0,01$ con $d=0$ y en la Figura $4(b)$ con factores de acoplamiento $v=0,1$ con valores de $d \neq 0$, los perfiles de dispersión son muy semejantes, y alcanzan valores equivalentes. Sin embargo, lo importante a detallar en estos casos de acoplamiento y en sus respectivos valores de " $d$ ", que pequeñas variaciones en la desintonización del haz de prueba, conllevan a cambios muy fuertes en la respuesta óptica, lo que permite 
suponer que estamos en presencia de un "suiche" óptico. Se nota en las propiedades dispersivas en la región de resonancia de prueba $\Delta_{2}\left(\mathrm{~s}^{-1}\right)=0$, su valor coincidente con el del solvente puro, (en este caso, agua) independientemente de la magnitud del factor de acoplamiento y de la diferencia de momentos dipolares permanentes. Las propiedades tanto absortivas como dispersivas mostradas, se corresponden con la misma transición de frecuencia entre estados adiabáticos. Un valor no nulo de la diferencia de momentos dipolares permanentes en los estados adiabáticos significa que por lo menos uno de los estados no tiene paridad definida, lo que se traduce en la presencia de elementos diagonales en el operador de momento dipolar. La existencia de estos elementos diagonales en el Hamiltoniano de interacción altera las propiedades ópticas no lineales de un sistema molecular en la intensidad de sus respuestas, y ocasiona la aparición de transiciones multifotónicas antes no permitidas, así como dispersión Raman o absorción de dos fotones. En moléculas polares como la empleada, el rol de estos momentos dipolares permanentes (Kmetic and Meath, 1985; Bavli et al., 1990; Jagatap \& Meath, 2002) es importante, dado que los mismos están involucrados en los mecanismos de excitación de dos fotones y cuantifican la transferencia de carga intramolecular durante la excitación electrónica (Jagatap \& Meath, 2002).

\section{CONCLUSIÓN}

La inserción del acoplamiento intramolecular en los tratamientos de respuestas ópticas no es muy común en la literatura. La forma del Hamiltoniano residual dependerá mucho de la naturaleza de la estructura molecular de estudio. Cuanto más acoplado sean estos movimientos electrónicos y nucleares, menor será la intensidad de la respuesta óptica. Estos estudios preliminares de consideración del acoplamiento intramolecular en todos los aspectos dinámicos de la matriz densidad, polarizaciones y susceptibilidades ópticas, permiten reconocer que en regiones de resonancia entre los haces incidentes del tratamiento MCO, conllevan a posibles construcciones de "suiches" ópticos en la propiedad refractiva, y de posibles 
amplificaciones paramétricas sintonizables con los colorantes orgánicos, en la propiedad absortiva. El resultado que mostramos en casos de nulidad de valores de " $d$ ", pueden referirse a la no existencia de momentos dipolares permanentes de la molécula en cada estado adiabático o que en ambos estados el valor de los mismos, sea exactamente igual.
Finalmente, las expresiones asociadas a los momentos dipolares de transición ecuación (4.a) y permanentes ecuación 4(b) en los estados adiabáticos, son de singular importancia para interpretaciones en los procesos de absorción de dos fotones y consideración explícita de la aproximación de onda rotante en los tratamientos del modelaje cuántico.

\section{LISTA DE REFERENCIAS}

Azumi, T. \& Matsuzaki, K. (1977). What does the term "vibronic coupling" mean? Photochemistry and Photobiology, 25(3), 315-326.

Bavli, R., Heller, D. \& Band, Y. (1990). Nonlinear optical properties of two-level systems with permanent dipole moments. Physical Review A. 41(7), 3960-3968.

Bersuker, I. (2006). The Jahn-Teller Effect. University Press Cambridge.

Boyd, R.W. (2008). Nonlinear optics: Academic Press, USA.

Castro, J., Yépez, E. \& Soto, J. (2004). No adiabaticidad en moléculas y el efecto JahnTeller. Revista Mexicana de Física 50(2), 123-131.

Christodoulides, D., Khoo, I., Salamo, G, Stegeman, G Van Stryland, E. (2010). Nonlinear refraction and absorption: mechanism and magnitudes. Advances in Optics and Photonics, 2(1), 60-200.

Cusati, T., Paz, J.L. \& Hernández, A. (2004). Effects of the dipole moments behavior on a vibronic coupling model for the Four-Wave Mixing signal. International Journal of Quantum Chemistry, 98(5), 425-433 
Di Bartolo., B y Foldberg, V. (1980). Radiationless Processes, Berlin, Springer.

Dunn, J., Alqannas, H. \& Lakin, A. (2015). Jahn-Teller effects and surface interactions in multiply-charged fullerene anions and the effect on scanning tunneling microscopy images. Chemical Physics, 460, 14-25.

Fulton, R. \& Gouterman, M. (1964). Vibronic coupling II. Spectra of dimers. The Journal of Chemical Physics, 41(8), 2280-2286.

García-Sucre, M., Goychman, F. \& Lefebvre, R. (1970). Adiabatic corrections in a simple model of two interacting electronic-potential curves. Physical Review A 2(5), 1738- 1745

Grochala, W. \& Hoffmann, R. (2000). Chemistry of vibronic coupling. How one might maximize off-diagonal dynamic vibronic coupling constants for intervalence charge-transfer (ivct) states in an aba system (a,b) alkali metal, h, halogen. The Journal of Physical Chemistry A, 104(43), 9740-9749

Guangjun, T. (2013) Electron- vibration coupling and its effects on optical and electronic properties of single molecule. PhD Thesis. Department of theoretical Chemistry and Biology, Royal Institute of Technology, UK

Guseinov, I., Mamedov, B. \& Ekenoglu, A. (2006). Analytical evaluation of two-center Franck-Condon overlap integrals over harmonic oscillator wave function. Journal of Physical Sciences, 61(3-4), 141-145.

Iwahara, N., Sato, T. \& Tanaka, K. (2011). Molecular design for high-spin molecules in view of vibronic couplings. Polyhedron, 30(18), 3048-3053.

Jagatap, B. \& Meath, W. (2002). Contributions of permanent dipole moments to molecular multiphoton excitation cross sections. Journal of the Optical Society of America $B, 19(11), 2673-2681$.

Kajzar, E, Charra, F., Nunzi, J., Raymond, P., Idiart., E. y Zagorska (1994). Third order nonlinear optical properties and functionalized polymers. En N. Paras (ED.) Frontiers of Polymers and Advanced Materials (11, pp 141-153). Berlin: Springer 
Kmetic, M. \& Meath, W. (1985). Permanent dipole moments and multiphoton resonances. Physics Letters A, 108(7), 340-343

Paz, J.L., García-Sucre, M., Squitieri, E. \& Mujica, V. (1994). The effect of intramolecular coupling on the optical susceptibilities of a two-level molecule. Chemical Physics Letters, 217(3), 333-341.

Paz, J.L., Mastrodomenico, A., Cárdenas, J., Rodríguez, L. y Costa-Vera, C. (2016a). En Lin, S.H., Villaeys, A.A. y Fujimura, Y. (ED). Advances in Multiphoton processes and Spectroscopy (23, pp 211-248). Londres: World Scientific Publishing.

Paz, J.L., Mastrodomenico, A., Costa-Vera, C., Cárdenas-García, J. \& Rodríguez, L.G (2015). Rotating wave approximation effects on the Nonlinear Optical responses of complex molecular systems Using a Four-Wave mixing signal. Journal of Modern Optics 62, 403-411.

Paz, J.L., Mastrodomenico, A. \& Izquierdo, M. (2013). Symmetry properties in the study of the Rayleigh-Type Optical Mixing signal in presence of a thermal bath. Journal of Nonlinear Optical Physics and Materials, 22(1), 1-13

Squitieri, E., García-Sucre, M., Paz, J.L. \& Mujica, V. (1994). Refractive index in a dilute solution of molecules with intramolecular coupling up to third order in the external field. Molecular Physics, 82(1), 227-234.

Teller, E. (1937). The crossing of potential surfaces. The Journal of Physical Chemistry, $431(1), 109-116$.

Walczak, K. (2006). The influence of vibronic coupling on the shape of transport characteristics in inelastic tunneling through molecules. Physica E: Low-dimensional Systems and Nanostructures, 33(1), 110-115. 